\title{
Comportamento térmico e evolução das fases de óxidos de Mn com estrutura hollandita da região Amazônica
}

\section{(Thermal behavior and evolution of the Mn oxides phases with hollandite structure from Amazon region)}

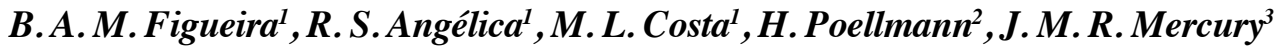 \\ ${ }^{1}$ Instituto de Geociências, Universidade Federal do Pará, R. Augusto Correa N-1, Belém, PA, Brasil 66075-110 \\ ${ }^{2}$ Instituto de Ciências Geológicas, Universidade Martin-Luther Wittenberg, Domstr. 6, D-06108, \\ Halle-Salle, Alemanha \\ ${ }^{3}$ Instituto Federal do Maranhão, Avenida Getúlio Vargas 4, Monte Castelo, S. Luís, MA, Brasil 65025-001 \\ bfigueira@ufpa.br
}

\begin{abstract}
Resumo
Minerais de óxidos de Mn com estrutura em túnel, hollandita (Apuí, Amazonas, Brasil, zona em prospecção) e criptomelana (Urucum, Mato Grosso do Sul, Brasil) foram isolados e caracterizados quanto à composição química, mineralógica, estabilidade térmica e morfologia. As seguintes técnicas foram utilizadas para caracterização: microscopia eletrônica de varredura-EDS, análise térmica (TG-DTA) e difração de raios X estático e com aquecimento contínuo entre 100-900 ${ }^{\circ} \mathrm{C}$. As seguintes fórmulas empíricas, calculadas com base em 16 átomos de oxigênios foram obtidas: $\left(\mathrm{Ba}_{0,18} \mathrm{~K}_{0,12} \mathrm{Ca}_{0,02} \mathrm{~Pb}_{0,04}\right)_{0,76}\left(\mathrm{Mn}_{6,34} \mathrm{Al}_{0,61} \mathrm{Si}_{0,25} \mathrm{Fe}_{0,24} \mathrm{Ti}_{0,08}\right)_{7,54} \mathrm{O}_{16} 0,4 \mathrm{H}_{2} \mathrm{O}$ para hollandita e $\left(\mathrm{K}_{0,9} \mathrm{Na}_{0,04} \mathrm{Ca}_{0,03} \mathrm{Sr}_{0,04}\right)_{1,04}\left(\mathrm{Mn}_{7,38} \mathrm{Fe}_{0,28} \mathrm{Al}_{0,27} \mathrm{Si}_{0,08}\right)_{8} \mathrm{O}_{16}$ para criptomelana. Mediante o uso de microscopia eletrônica de varredura foi possível diferenciar a morfologia da hollandita e da criptomelana. Os resultados de DRX e TG-DTA mostraram que os minerais apresentaram estabilidade térmica acima de $900{ }^{\circ} \mathrm{C}$.
\end{abstract}

Palavras-chave: hollandita, criptomelana, Apuí, Urucum, caracterização mineral.

\section{Abstract}

The tunnel-structure manganese oxide minerals, hollandite (Apuí, Amazonas state, Brazil, area in prospection) and cryptomelane (Urucum, Mato do Sul Grosso state, Brazil) were isolated and characterized chemical, mineralogical, thermal and morphologically. The following techniques were employed: scanning electron microscopy-EDS, thermal analysis (DTA-TG), X-ray diffraction with continuous-heating. The empirical formula, were calculated on the basis 16 oxygen gave as $\left(\mathrm{Ba}_{0.18} \mathrm{~K}_{0.12} \mathrm{Ca}_{0.02} \mathrm{~Pb} 0.04\right)_{0.76}\left(\mathrm{Mn}_{6.34} \mathrm{Al}\right.$ $\left.{ }_{0.61} \mathrm{Si}_{0.25} \mathrm{Fe}_{0.24} \mathrm{Ti}_{0.08}\right)_{7.54} \mathrm{O}_{16} \mathrm{O} .4 \mathrm{H}_{2} \mathrm{O}$ for hollandite and $\left(\mathrm{K}_{0.9} \mathrm{Na}_{0.04} \mathrm{Ca}_{0.03} \mathrm{Sr}_{0.04}\right)_{1.04}\left(\mathrm{Mn}_{7.38} \mathrm{Fe}_{0.28} \mathrm{Al}_{0.27} \mathrm{Si}_{0.08}\right)_{8} \mathrm{O}_{16}$ for cryptomelane. The SEM investigations allowed the differentiation of the morphologies for hollandite and cryptomelane. The minerals were thermally treated among $100-900{ }^{\circ} \mathrm{C}$ and $X$-ray diffraction patterns in situ were collected. The analysis of $X$-ray diffractions experiments showed that a hollandite-crytomelane mineral has a thermal stability up to $900{ }^{\circ} \mathrm{C}$.

Keywords: hollandite, cryptomelane, Apuí, Urucum, mineral characterization.

\section{INTRODUÇÃO}

O grupo da hollandita consiste de um grande número de minerais cujos óxidos possuem uma estrutura em túnel formada a partir de octaedros ligados entre si pelas arestas e vértices. Sua fórmula teórica é $A_{x} B_{8} O_{16}(0 \leq x \leq 2)$, em que A representa os túneis parcialmente preenchidos por cátions mono e divalentes $\left(\mathrm{K}^{+}, \mathrm{Na}^{+}, \mathrm{Ba}^{2+}, \mathrm{Pb}^{2+}, \mathrm{Sr}^{2+}\right)$, enquanto $\mathrm{B}$ corresponde a os sítios octaédricos onde podem estar presentes os cátions $\mathrm{Cr}^{3+}, \mathrm{Fe}^{3+}, \mathrm{V}^{3+} \mathrm{Mn}^{4+}, \mathrm{Ti}^{4+}, \mathrm{Zr}^{4+},[1,2]$. No caso dos óxidos de Manganês, a estrutura da hollandita é caracterizada por cátions $\mathrm{Mn}^{4+}$ nos octaedros em um arranjo $2 \times 2$, com adicionais substituições desta espécie por $\mathrm{Mn}^{3+}, \mathrm{Si}^{4+}, \mathrm{Ti}^{4+}$ e $\mathrm{Al}^{3+}$ as quais geram um déficit de carga na estrutura, que é compensada pela presença de cátions mono e divalentes (Fig. 1), como ocorrem com os minerais de hollandita (bário), criptomelana (potássio), coronadita (chumbo), manjiroita (sódio) e estrônciomelana (estrôncio) $[3,4]$.

Em ambientes naturais, estes compostos são importantes na geoquímica de metais e não metais, em virtude de sua capacidade em oxidar íons $\mathrm{Co}^{2+}, \mathrm{Cr}^{3+}, \mathrm{As}^{3+}$ para $\mathrm{Co}^{3+}, \mathrm{Cr}^{6+}$, $\mathrm{As}^{5+}$, e influenciar na mobilidade e toxidade destes elementos em solos, sedimentos, depósitos marinhos e terrestres. Eles também participam em atividades catalíticas para a formação de substâncias húmicas em ambientes abióticos [5, 6]. Devido às propriedades físico-químicas dos minerais do grupo da hollandita, diversos processos de síntese têm sido desenvolvidos com o intuito de obter peneiras moleculares conhecidas como OMS-2 (octahedral molecular sieves) com alta estabilidade térmica e propriedades especiais de troca iônica, sorção e redox [7-9].

No presente estudo descreve pela primeira vez a ocorrência de hollandita em Apuí (estado do Amazonas, 


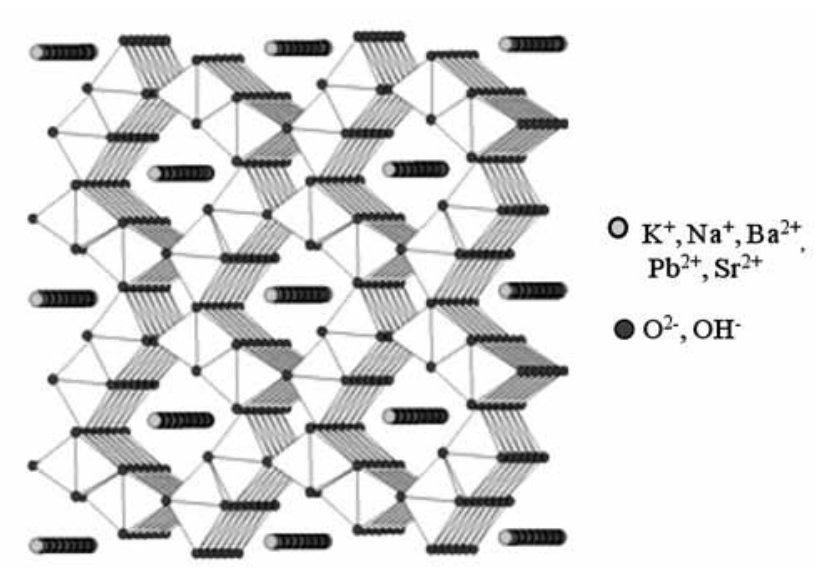

Figura 1: Ilustração da estrutura hollandita.

[Figure 1: Illustration of the hollandite structure.]

área em prospecção). Também se descreve a caracterização detalhada de criptomelana, obtida da famosa mina de Mn de Urucum (Mato Grosso do Sul).

\section{MATERIAIS E MÉTODOS}

Preparação das amostras: as fases em estudo (criptomelana e hollandita) foram isoladas dos minérios de óxidos de Mn provenientes da mina do Urucum (Mato Grosso do Sul) e Apuí (Amazonas, área em prospecção) e codificadas como UR-01 e AP-01, respectivamente. Os minerais foram isolados usando-se pinça de aço inox com o auxilio de um microscópio binocular (Carl Zeiss). Uma pequena quantidade de cada amostra foi cuidadosamente pulverizada em um grau de ágata. O pó obtido foi misturado com acetona e depositado num porta-amostra de silício para formar um fino filme do mineral para posterior análise por difração de raios $\mathrm{X}$.

Análise térmica: as curvas de TG e DTA foram obtidas num termoanalisador Stanton Redcroft de forno cilíndrico vertical, usando-se como porta mostra um cadinho de platina, a uma taxa de aquecimento de $15{ }^{\circ} \mathrm{C} / \mathrm{min}$, no intervalo 25 $1100{ }^{\circ} \mathrm{C}$.

Morfologia: para os estudos de microscopia eletrônica de varredura (MEV) e micro-análise por EDS (4 pontos), as amostras foram pulverizadas e dispersas em suportes impregnados com grafite e metalizadas com ouro. O instrumento utilizado foi um microscópio LEO Zeiss 430 Vp, em condições de análise utilizando imagens secundárias obtidas a $20 \mathrm{kV}$, com distância de trabalho de $11 \mathrm{~mm}$.

Experimentos de difração de raios $X(D R X)$ : as análises foram realizadas num difratômetro X'Pert Pro MPD (PW 3040/60) PANalytical, com goniômetro PW3050/60 (Teta/ Teta) e com tubo de raios $\mathrm{X}$ cerâmico de anodo de Co (ka1 $1,79 \AA$ A), foco fino longo, $2200 \mathrm{~W}, 60 \mathrm{kV}$, com filtro $\mathrm{k}_{\beta}$ de Ni.O detector utilizado é do tipo RTMS, X'Celerator. A aquisição de dados foi feita com o software X'Pert Data Collector 2.1a, e o tratamento dos dados com o software $\mathrm{X}^{\prime}$ Pert HighScore 3.0. Para o monitoramento das transformações de fases por DRX in situ, foi utilizado um difratômetro
X'Pert Pro MPD PW 3373/00 com tubo de raios X cerâmico de anodo de $\mathrm{Cu}(\mathrm{k} \alpha, 1,540598 \AA$ A $)$, equipado com câmara de alta temperatura Anton Parr modelo HTK16 com portaamostra de Pt. As seguintes condições experimentais foram empregadas: taxa de aquecimento $5{ }^{\circ} \mathrm{C} / \mathrm{min}$, com tempo de patamar $5 \mathrm{~min}$, na faixa de temperatura $100-900{ }^{\circ} \mathrm{C}$, no intervalo angular $10-55^{\circ}(2 \theta)$, passo: $0,017^{\circ}(2 \theta)$, tempo/ passo $29 \mathrm{~s} /{ }^{\circ}$, com resolução temporal de $7 \mathrm{~min}$. Em razão da temperatura dos experimentos descritos no anteriormente não ter ultrapassado $900^{\circ} \mathrm{C}$, amostras do material em estudo foram tratadas termicamente a 950 e $1050{ }^{\circ} \mathrm{C}$ em cadinho de porcelana num forno elétrico convencional a uma taxa de aquecimento de $5^{\circ} \mathrm{C} / \mathrm{min}$ e $1 \mathrm{~h}$ de patamar. $\mathrm{O}$ material resultante $(15 \mathrm{mg})$ foi utilizado para estudo por DRX em modo convencional cujos registros foram coletados nas seguintes condições: $40 \mathrm{kV}, 40 \mathrm{~mA}$, faixa de varredura 5-70 $(2 \theta)$, passo $0,017^{\circ}(2 \theta)$ e tempo de contagem $60 \mathrm{~s}$, fenda divergente $1 / 8^{\circ}$ e anti-espalhamento de $1 / 4^{\circ}$, máscara de 10 $\mathrm{mm}$, com rotação do porta mostra.

Análise química: a determinação dos elementos maiores ( $\mathrm{Mn}, \mathrm{K}, \mathrm{Fe}, \mathrm{Ba}$ e $\mathrm{Al}$ ) nas amostras em estudo foi determinada com base na ref. [4], em que foi obtida a composição química dos minerais de Mn através de micro-análise por SEM-EDS. Os elementos maiores e traços presentes nas amostras em baixa quantidade foram determinados por ICPOES (Plasmaquant 110). O teor de $\mathrm{Mn}_{2} \mathrm{O}_{3}$ foi calculado de acordo com a ref. [10].

\section{RESULTADOS E DISCUSSÃO}

Os difratogramas de raios $\mathrm{X}$ das amostras UR-01 e AP01 são mostrados na Fig. 2.

Todas as reflexões do padrão DRX de UR-01 observadas na Fig. 2a podem ser diretamente indexadas a criptomelana, fase tetragonal (grupo espacial $\mathrm{I} 4 / \mathrm{m}$ ), com parâmetros de cela unitária ( $a=9,80 \AA$ e $c=2,85 \AA$ ), compatíveis com os dados reportados no arquivo PDF 29-1020. Os picos mais intensos presentes a $d=6,92,4,89,3,46,3,10,2,39$, 2,15, 1,82 e 1,53 Å correspondem aos planos da estrutura de criptomelana (110), (200), (310), (211), (310), (410), (521), respectivamente. Já a amostra AP-01 (Fig. 2b), apresenta reflexões que sugerem a presença de hollandita monoclínica, com grupo espacial $\mathrm{I} 2 / \mathrm{m}$, parâmetros de cela ( $a=10,90$ $\AA ; b=2,85 \AA ; c=9,60 \AA ; \beta=91,72^{\circ}$ ). Estes resultados corroboram aqueles descritos no arquivo PDF 38-0476. Os oito picos mais intensos com espaçamentos $d$ de 6,86 , $4,84,3,47,3,11,2,40,2,15$ e $1,54 \AA$ de AP-01 podem ser diretamente indexados aos planos (110), (200), (220), (310), (410), (301), (520) e (521), respectivamente. A presença dos picos correspondentes aos planos (110), (200) e (310) é um dos principais indicativos da presença deste mineral [11]. A intensidade do pico referente ao plano (200) na amostra AP01, mais intensa do que observado no padrão PDF (Fig. 2b) ocorre em função da orientação preferencial de estruturas nanofibrosas como hollandita [12].

As análises elementares das amostras indicam que as mesmas são compostas predominantemente por $\mathrm{Mn}$, com 


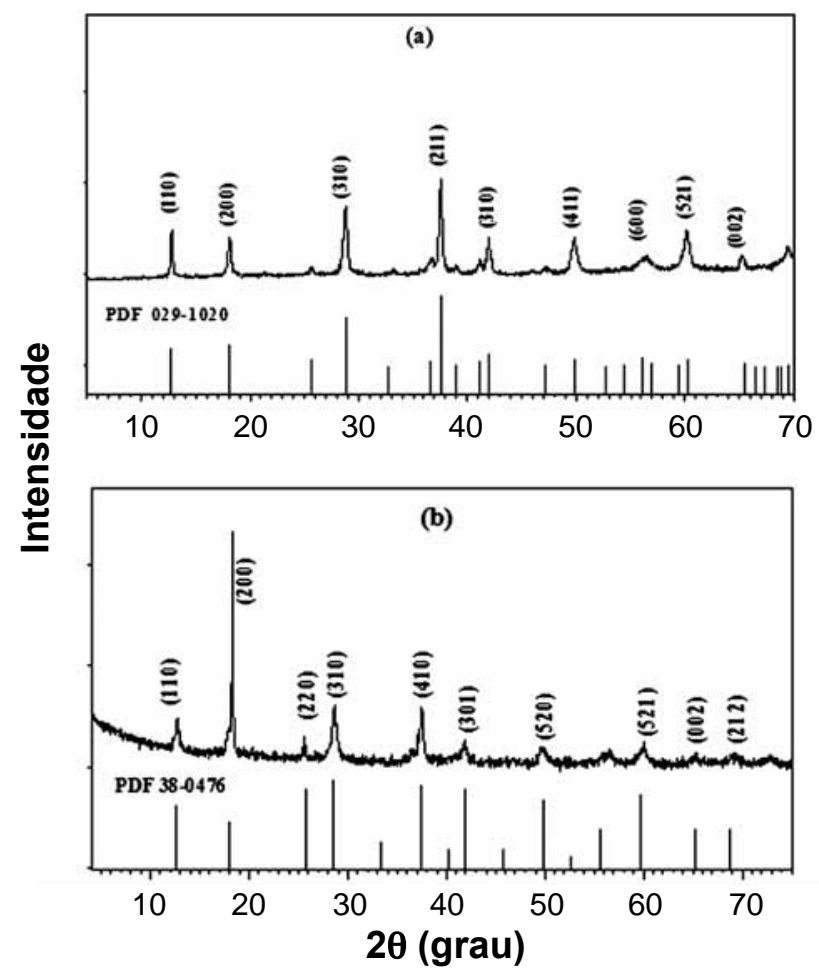

Figura 2: Difratograma de raios X de UR-01 (a) e AP-01 (b). [Figure 2: XRD patterns of UR-01 (a) and AP-01 (b).]

teor total (em forma de óxido) acima de $80 \%$ em UR-01 e $75 \%$ em AP-01 (Tabela I). Outros elementos também estão presentes em menor quantidade, com destaque para $\mathrm{K}, \mathrm{Fe}$ e Al na amostra UR-01 e Ba, Al, Fe, Si, Ti, K e Pb na amostra AP-01.

Com base nos resultados da analise química (Tabela I) e de acordo com os estudos de Post et al. (1982) e Post e Burnham (1986) [1, 13], pode-se afirmar que os cátions presentes nos túneis de criptomelana em estudo são predominantemente $\mathrm{K}^{+}$, embora haja a presença em pequena quantidade de $\mathrm{Na}^{+}, \mathrm{Ca}^{2+}$ e $\mathrm{Sr}^{2+}$. O total de íons nos tuneis de criptomelana está em torno de 1,04 que

Com base nos resultados da analise química (Tabela I) e de acordo com estudos [1, 13], pode-se afirmar que os cátions presentes nos túneis de criptomelana são predominantemente $\mathrm{K}^{+}$, embora haja pequena quantidade de $\mathrm{Na}^{+}, \mathrm{Ca}^{2+} \mathrm{e} \mathrm{Sr}^{2+}$. O total de íons nos tuneis de criptomelana está em torno de 1,04 que corresponde a 52\%, valor acima da média de ocupação (45\%) para estruturas deste tipo, que se atribui provavelmente a íons adsorvidos à superfície do material $[10,14]$. Em relação a hollandita observa-se que a soma dos cátions na estrutura $(0,76)$ equivale a $38 \%$ dos túneis, com predomínio de $\mathrm{Ba}^{2+}$ e $\mathrm{K}^{+}$numa razão de 3:2, embora também ocorra solução sólida dos íons $\mathrm{Ba}^{2+}, \mathrm{Ca}^{2+} \mathrm{e}$ $\mathrm{Pb}^{2+}$. A estrutura possui aproximadamente 0,4 moléculas de água que preenchem alguns dos sítios não ocupados pelos cátions na estrutura [15]. A fórmula química dos minerais, com base nos resultados da Tabela I, pode ser expressa da seguinte forma:

$\left.\mathrm{Ba}_{0,18} \mathrm{~K}_{0,12} \mathrm{Ca}_{0,02} \mathrm{~Pb}_{0,04}\right)_{0,76}\left(\mathrm{Mn}_{6,34} \mathrm{Al}_{0,61} \mathrm{Si}_{0,25} \mathrm{Fe}_{0,24} \mathrm{Ti}_{0,08}\right)_{7,54} \mathrm{O}_{16} 0,4 \mathrm{H}_{2} \mathrm{O}$
Tabela I - Composição química de UR-01 e AP-01. Os elementos estão em \% de peso.

[Table I - Chemical composition (wt. \%) of UR-01 and AP01.]

\begin{tabular}{|c|c|c|}
\hline Óxidos & $\begin{array}{c}\text { Criptomelana } \\
\text { (UR-01) }\end{array}$ & $\begin{array}{c}\text { Hollandita } \\
\text { (AP-01) }\end{array}$ \\
\hline $\mathrm{SiO}_{2}$ & 0,69 & 2,43 \\
\hline $\mathrm{TiO}_{2}$ & 0,12 & 1,16 \\
\hline $\mathrm{Al}_{2} \mathrm{O}_{3}^{2}$ & 1,91 & 5,17 \\
\hline $\mathrm{Fe}_{2} \mathrm{O}_{3}$ & 3,12 & 3,21 \\
\hline $\mathrm{MnO}_{2}$ & 78,08 & 53,52 \\
\hline $\mathrm{Mn}_{2} \mathrm{O}_{3}$ & 8,69 & 24,31 \\
\hline $\mathrm{CaO}$ & 0,27 & 0,21 \\
\hline $\mathrm{MgO}$ & 0,01 & $<0,01$ \\
\hline $\mathrm{Na}_{2} \mathrm{O}$ & 0,18 & $<0,01$ \\
\hline $\mathrm{K}_{2}^{2} \mathrm{O}$ & 5,78 & 1,03 \\
\hline $\mathrm{BaO} \cong$ & 0,29 & 4,81 \\
\hline $\mathrm{ZnO} \frac{\pi}{0}$ & 0,14 & $<0,33$ \\
\hline $\mathrm{SrO} \bar{a}$ & 0,59 & $<0,05$ \\
\hline $\mathrm{PbO} \stackrel{\text { Ф }}{ \pm}$ & $<0,01$ & 1,37 \\
\hline $\mathrm{H}_{2} \mathrm{O} \cong$ & - & 7,04 \\
\hline Total & 99,88 & 101,01 \\
\hline \multicolumn{3}{|c|}{ Num. Cátions na base de 16 Oxigênios } \\
\hline $\mathrm{Mn}^{4+}$ & 6,58 & 3,73 \\
\hline $\mathrm{Ti}^{4+}$ & 0,01 & 0,08 \\
\hline $\mathrm{Si}^{4+}$ & 0,08 & 0,25 \\
\hline $\mathrm{Mn}^{3+}$ & 0,80 & 2,61 \\
\hline $\mathrm{Al}^{3+}$ & 0,27 & 0,61 \\
\hline $\mathrm{Fe}^{3+}$ & 0,28 & 0,24 \\
\hline \multicolumn{2}{|c|}{ Total (octaedros) 8,02} & 7,54 \\
\hline $\mathrm{K}+$ & 0,90 & 0,12 \\
\hline $\mathrm{Na}+$ & 0,04 & $<0,01$ \\
\hline $\mathrm{Ca}^{2+}$ & 0,03 & 0,02 \\
\hline $\mathrm{Mg}^{2+}$ & 0,01 & $<0,01$ \\
\hline $\mathrm{Ba}^{2+}$ & 0,01 & 0,18 \\
\hline $\mathrm{Sr}^{2+}$ & 0,04 & $<0,01$ \\
\hline $\mathrm{Pb}^{2+}$ & $<0,01$ & 0,04 \\
\hline $\mathrm{Zn}^{2+}$ & 0,01 & $<0,01$ \\
\hline $\mathrm{H}_{2} \mathrm{O}$ & - & 0,4 \\
\hline Total (túr & 1,04 & 0,76 \\
\hline
\end{tabular}

para hollandita e $\left(\mathrm{K}_{0,9} \mathrm{Na}_{0,04} \mathrm{Ca}_{0,03} \mathrm{Sr}_{0,04}\right)_{1,04}\left(\mathrm{Mn}_{7,38} \mathrm{Fe}_{0,28} \mathrm{Al}_{0,27} \mathrm{Si}_{0,08}\right)_{8} \mathrm{O}_{16}$ para criptomelana.

A Fig. 3 apresenta as curvas TG-DTA de UR-01 (Fig. 3a) e AP-01 (Fig. 3b).

Nesta se observa que ambas as amostras apresentam três reações endotérmicas. A primeira na faixa entre 625 e $650{ }^{\circ} \mathrm{C}$ com perda de massa $4,5 \%$ para UR-01 e $4,80 \%$ 


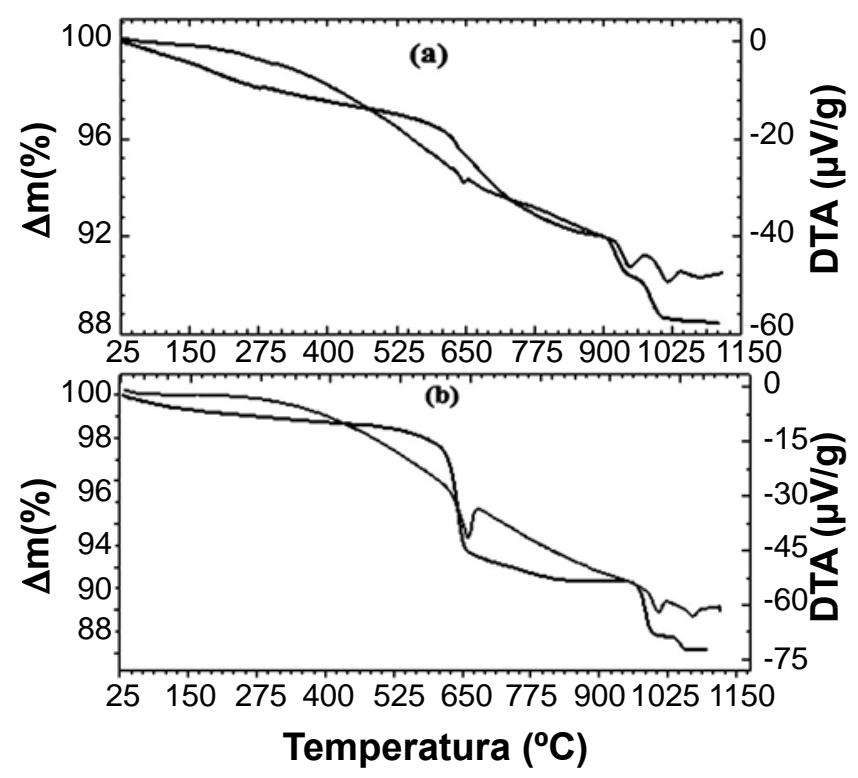

Figura 3: Curvas TG/DTA a $15^{\circ} \mathrm{C} / \mathrm{min}$ de UR-01(a) e AP-01 (b). [Figure 3: TG-DTA curves at $15^{\circ} \mathrm{C} / \mathrm{min}$ of UR-01 (a) and AP-O1 (b).]

para AP-01, que se atribui à redução parcial de Mn (IV) em $\mathrm{Mn}$ (III) nos octaedros $\mathrm{MnO}_{6}$ com perda de oxigênio do retículo (Eq.A), com a formação de vazios e transformação do sistema cristalino tetragonal para monoclínico, da fase criptomelana $[10,11,16]$, de acordo com seguinte equação:

$$
\mathrm{Mn}^{4+}+\mathrm{O}_{\text {(retículo) }}^{2-} \rightarrow \mathrm{Mn}^{3+}+1 / 2 \mathrm{O}+\mathrm{V}_{0 \text { (vacâncias de oxigênio) }}
$$

O segundo pico endotérmico próximo a $950{ }^{\circ} \mathrm{C}$, com perda de massa aproximada de $2,48 \%$ para UR-01 e $2,57 \%$
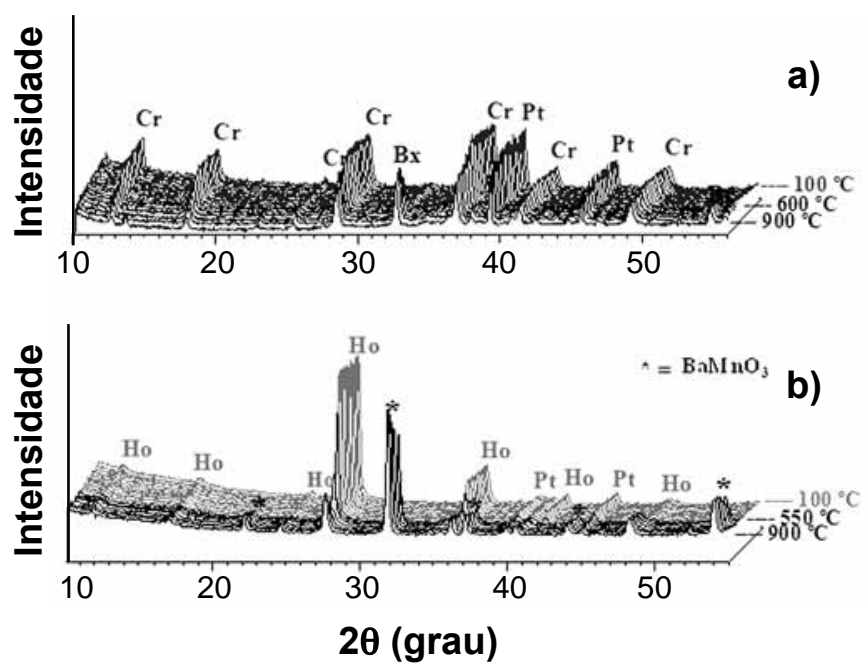

Figura 4: Mudanças nos padrões DRX das amostras entre 100 e $900{ }^{\circ} \mathrm{C}$ para UR-01 (a) e AP-01 (b) em condições experimentais dinâmicas. $(\mathrm{Cr}=$ criptomelana, $\mathrm{Ho}=$ hollandita, $\mathrm{Bx}=$ bixbyita, $\mathrm{Pt}=$ platina).

[Figure 4: Changes of XRD pattern of the samples UR-01 (a) and AP-01 (b) heated in the $100-900{ }^{\circ} \mathrm{C}$ range under dynamic experimental conditions. (Cr=criptomelane, Bx=bixbyite, Ha=hausmannite, $P t=$ platinum $)$.] para AP-01 é comum em amostras contendo minerais do grupo da hollandita, e está relacionada à transformação destas fases para bixbyita, $\mathrm{Mn}_{2} \mathrm{O}_{3}$. O último pico acima de $1000{ }^{\circ} \mathrm{C}$, com perda de $1,76 \%$ (UR-01) e $0,56 \%$ (AP$01)$, indicam uma incompleta conversão de $\mathrm{Mn}_{2} \mathrm{O}_{3}$ em $\mathrm{Mn}_{3} \mathrm{O}_{4}$ (perda de massa teórica 3\%) [17]. O comportamento térmico e a evolução das fases criptomelana e hollandita com a temperatura (100-900 $\left.{ }^{\circ} \mathrm{C}\right)$ na amostra UR-01 e AP-01 respectivamente, foram monitoradas por difração de raios $\mathrm{X}$ in situ, conforme pode ser observado nas Figs. 4a-b.

Na Fig. 4a é possível observar a manutenção da fase criptomelana no intervalo $100-550{ }^{\circ} \mathrm{C}$ sem danos na estrutura. A partir de $600{ }^{\circ} \mathrm{C}$ ocorre a nucleação da fase bixbyita $\left(\mathrm{Mn}_{2} \mathrm{O}_{3}\right)$ em $33^{\circ}(2 \theta)$, que aumenta de intensidade enquanto os picos principais de criptomelana a 28 e $37^{\circ}(2 \theta)$ decrescem consideravelmente até $900{ }^{\circ} \mathrm{C}$. Estes resultados revelam um intenso processo de perda de manganês dos octaedros de criptomelana, e consequentemente, oxidação dos mesmos com a formação de $\mathrm{Mn}_{2} \mathrm{O}_{3}$, corroborando com os dados TG-DTA anteriormente discutidos. Em relação à fase hollandita, a estrutura se mantém estável até $500{ }^{\circ} \mathrm{C}$, Fig. 4b. A $550{ }^{\circ} \mathrm{C}$ se verifica o decréscimo do pico principal de hollandita a $28^{\circ}(2 \theta)$ que se estende até $900^{\circ} \mathrm{C}$, enquanto um pico referente a fase $\mathrm{BaMnO}_{3}$ (PDF 026-0167) é

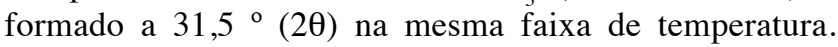
Com base nestas informações, pode-se afirmar que a partir de $550{ }^{\circ} \mathrm{C}$, cátions $\mathrm{Ba}^{2+}$ são liberados dos túneis, reagem com todos os cátions $\mathrm{Mn}$ (ausência de $\mathrm{Mn}_{2} \mathrm{O}_{3}$ ) liberados dos octaedros para formar a fase $\mathrm{BaMnO}_{3}$ de tipo espinélio.

Para complementar os estudos por DRX, os difratogramas estáticos de criptomelana (UR-01) e hollandita (AP-01) aquecidas a 950 e $1050{ }^{\circ} \mathrm{C}$ foram coletados e são mostrados nas Figs. 5a-b.
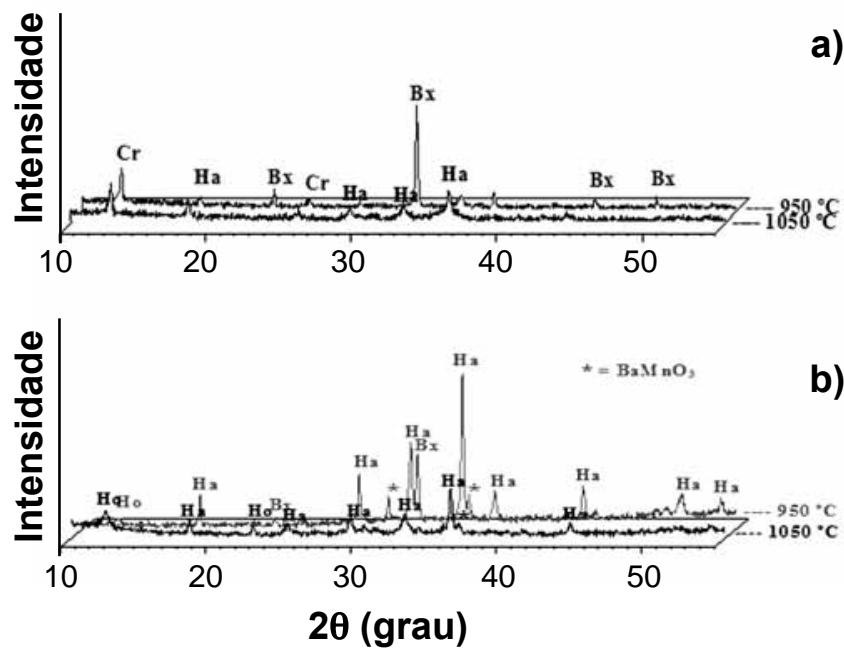

Figura 5: Mudanças nos padrões DRX das amostras a 950 e $1050^{\circ} \mathrm{C}$ para UR-01 (a) e AP-01 (b) em condições experimentais estáticas. $(\mathrm{Cr}=$ criptomelana, $\mathrm{Ho}=$ hollandita, $\mathrm{Bx}=$ bixbyita, $\mathrm{Ha}=$ hausmannita, $\mathrm{Pt}=$ Platina).

[Figure 5: Changes of XRD patterns of the samples UR-01 (a) and $A P-01$ (b) heated at 950 and $1050{ }^{\circ} \mathrm{C}$ under static experimental conditions. (Cr=cryptomelane, Bx=bixbyite, Ha=hausmannite, $P t=$ platine).] 

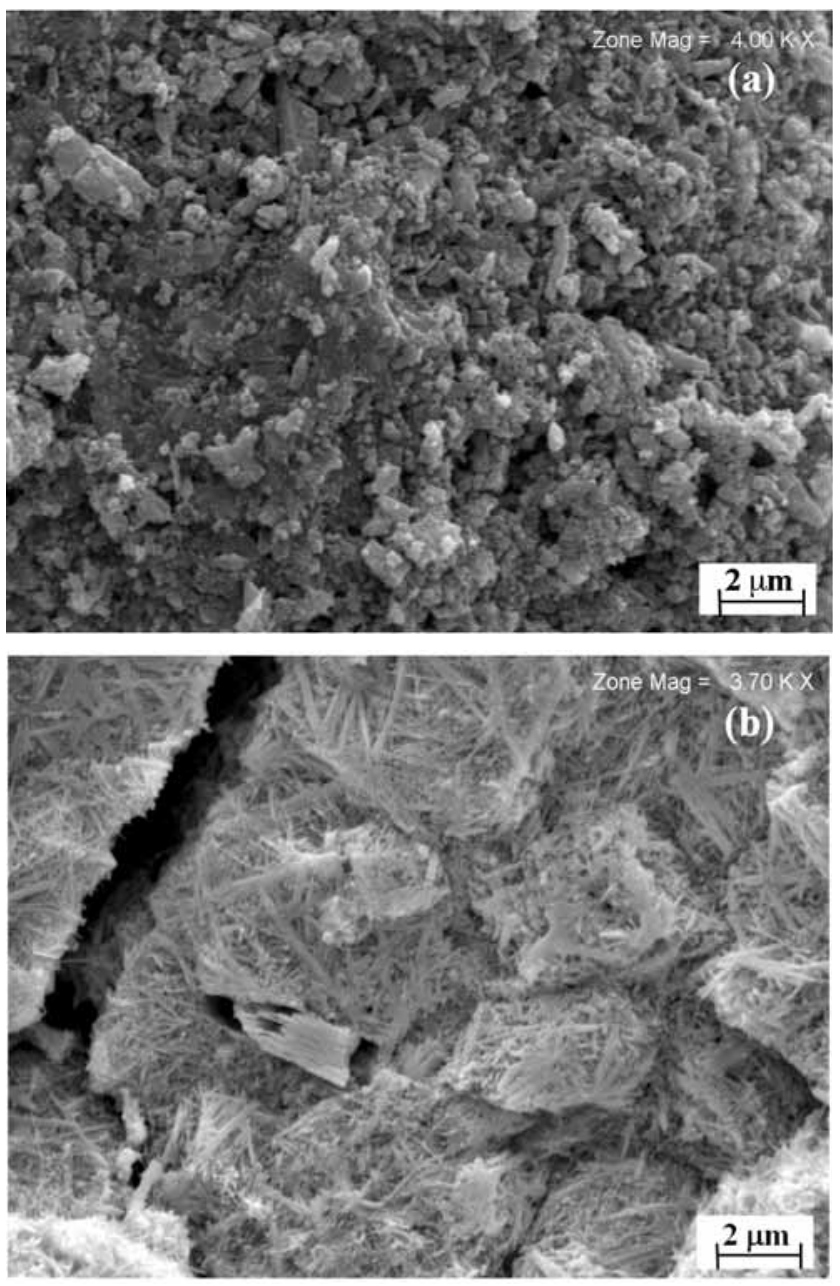

Figura 6: Fotomicrografia de microscopia eletrônica de varredura de (a) UR-01 e (b) AP-01.

[Figure 6: Scanning electron microscopy micrographs of (a) UR01 and (b) AP-01.]

A análise dos experimentos estáticos para criptomelana (Fig. 5a) mostra que a bixbyita, formada a $600{ }^{\circ} \mathrm{C}$ permanece estável até $950{ }^{\circ} \mathrm{C}$; porém nesta temperatura começa a decompor para formar a fase $\mathrm{Mn}_{3} \mathrm{O}_{4}$, conversão que se estende até 1050 . Acima de $1050{ }^{\circ} \mathrm{C}$, ainda é possível observar picos residuais de criptomelana, o que demonstra que $0,9 \%$ dos cátions $\mathrm{K}^{+}$dentro dos túneis, revelados na análise química, estabilizam a rede mantendo a estrutura acima desta temperatura. Já a hollandita mostra comportamento térmico similar a criptomelana, com a manutenção da estrutura acima de $1000^{\circ} \mathrm{C}$, como se observa pela presença do pico próximo a $11^{\circ}(2 \theta)$, Fig. $5 \mathrm{~b}$. Conforme revelado nas análises químicas de AP-01, a presença de $0,76 \%$ de cátions dentro dos túneis ajuda a manter a estrutura a $1050^{\circ} \mathrm{C}$.

A Fig. 6 exibe as imagens de microscopia eletrônica de varredura das amostras UR-01 (criptomelana) e AP01 (hollandita). As morfologias indicam que UR-01 se apresenta na forma de aglomerados de criptomelana não uniformes, resultado que contrasta com os cristais aciculares de criptomelana da mina do Azul, descritos e reportados [18]. Já a amostra (AP-01) revela uma morfologia composta de aglomerados de agulhas nanométricas de hollandita, bastante similar aos resultados reportados [19-21].

\section{CONCLUSÃO}

Os minerais hollandita e criptomelana foram isolados com sucesso por um método simples de micropreparação. Os resultados por micro-análise indicam a presença majoritária de cátions $\mathrm{K}^{+}$nos túneis de criptomelana e $\mathrm{Ba}^{2+} \mathrm{em}$ hollandita, embora se observe soluções sólidas em pequenas quantidades com cátions $\mathrm{Na}^{+}, \mathrm{Ca}^{2+}, \mathrm{Sr}^{2+} \mathrm{e} \mathrm{Pb}^{2+}$. Em relação ao comportamento térmico, os minerais apresentam estabilidade térmica acima de $1000{ }^{\circ} \mathrm{C}$. Em torno de $550{ }^{\circ} \mathrm{C}$, os cátions $\mathrm{Mn}^{3+4+} \mathrm{e} \mathrm{Ba}^{2+}$ são gradativamente removidos dos túneis, sofrem oxidação e formam as fases $\mathrm{Mn}_{2} \mathrm{O}_{3}$ (criptomelana) e $\mathrm{BaMnO}_{3}$ (hollandita), respectivamente. Criptomelana se apresenta em forma de glóbulos não uniformes, enquanto hollandita em agulhas.

\section{AGRADECIMENTOS}

Os autores agradecem ao CNPq pelo auxilio financeiro: bolsa de doutorado do primeiro autor (Proc. 141047/20089) e pela bolsa de produtividade em pesquisa do segundo autor (Procs: 304.566/2007-1 e 303.871/2010-5), e ao Projeto MINOMAT - Dos minerais aos novos materiais: Caracterização de matérias-primas e rejeitos minerais, modificação, síntese e aplicações industriais. Ed. 02/2007 Seleção de Projetos de Pesquisa do Programa de Apoio a Núcleos de Excelência (PRONEX) da FAPESPA e do CNPq.

\section{REFERÊNCIAS}

[1] J. E. Post, C. W. Burnham, Amer. Miner. 71 (1986) 1178. [2] M. Pasero, Rev. Miner. Geochem. 57 (2005) 291.

[3] R. G. Burns, V. M. Burns, Marine Manganese Deposits, $1^{\text {st }}$ Ed., Elsevier Oceanography Series, Amsterdam, Holanda (1979) 188.

[4] N. Meisser, E-A Perseil, J. Brugger, P-J. Chiappero, Canad. Miner. 37 (1999) 673.

[5] S. M. Webb, B. M. Tebbo, J. R. Bargar, Am. Miner. 90 (2005) 1342.

[6] D. Hillel, Encyclopedia of soils in the environment, $1^{\text {st }}$ Ed., Academic Press, New York, EUA (2004) 433.

[7] S. L. Brock, N. Duan, Z. R. Tian, O. Giraldo, H. Zhou, S. L. Suib, Chem. Mater. 10 (1998) 2619.

[8] Q. Feng, K. Yanagisawa, K. Yamasaki, J. Porous Mater. 5 (1999) 153.

[9] Q. Feng, H. Kanoh, K. Ooi, J. Mater. Chem. 9 (1999) 319.

[10] H. Kudo, H. Miura, Y. Hariya, Miner. J. 15 (1990) 50.

[11] Q. Zhang, S. L. Suib, Chem. Mater. 11 (1999) 1306.

[12] W-N. Li, J. Yuan, S. Gomez, S. L. Suib, J. Phys. Chem. B 110 (2006) 3066.

[13] J. E. Post, R. B. Dreele, P. R. Buseck, Acta Cryst. B 38 (1982) 1056.

[14] M. Nambu, K. Tanida, J. Miner. Soc. Japan 14 (1980) 
62.

[15] A. Byström, A. M. Byström, Acta Cryst. 3 (1950) 146. [16] X. Yang, Y. Makita, Z-H, Liu, K. Sakane, K. Ooi, Chem. Mater. 16 (2004) 5581.

[17] D. L. Bish, J. E. Post, Am. Miner. 74 (1989) 177.

[18] M. L. Costa, O. J. C. Fernandez, M. E. R. Requelme, Caracterização de depósitos minerais em distritos Mineiros da Amazônia, Brasilia: DNPM-CT/Mineral-ADIMB (2005)
227.

[19] S. Ching, E. J. Welch, S. M. Hughes, A. B. F Bahadoor, Chem. Mater. 14 (2002) 1292.

[20] Y-S. Ding, X-F. Shen, S. Sithambaram, S. L. Suib, Chem. Mater. 17 (2005) 5382.

[21] R. Jothiramalingar, B. Viswanathan, T. K. Varadarajan, Mater. Chem. Phys. 100 (2006) 257.

(Rec. 11/02/2012, Ac. 21/04/2012) 